\title{
MSC - a Multipotent Stromal Cell in Search of Clinical Application
}

\author{
Torsten Tonn ${ }^{\mathrm{a}} \quad$ Dagmar Barz ${ }^{\mathrm{b}}$ \\ ${ }^{a}$ Institute for Transfusion Medicine and Immunohematology, Clinics of the Johann Wolfgang Goethe University Frankfurt/Main, \\ Red Cross Blood Donor Service Baden-Württemberg-Hessen, Frankfurt/M. \\ b Institute for Transfusion Medicine, Friedrich Schiller University Clinics Jena, Germany
}

As pointed out by the International Society for Cellular Therapy (ISCT) position statement [1], the term MSC describes a cell population of plastic-adherent multipotent mesenchymal stromal cells, comprising a subset of cells with defined stem cell activity (i.e. the ability to undergo self-renewal or asymmetric cell division), which is synonymously also referred to as mesenchymal stem cells. By virtue of their multipotency, which allows differentiation into muscle, bone, cartilage or adipose tissue, the potential clinical applications for MSCs are manifold. In addition, MSCs have been shown to negatively modulate immune responses and to specifically home into cancer tissues, which makes them potential candidates as cellular vehicles in certain immunological abnormalities, such as graftversus-host disease (GvHD) and autoimmune diseases, and in the treatment of solid cancers, respectively. While these almost unlimited clinical perspectives have attracted many groups to MSC research, MSC therapy has not yet achieved standard of care status for any medical condition. In fact, the transfer of MSC-based therapies from bench to bedside is hampered by several factors which need to be addressed carefully in order to provide safe therapies which manage to exploit the therapeutic potential of MSCs. In this issue, several leaders in the field review important issues that need to be addressed when striving to successfully develop MSC-based therapies from bench to bedside. Since MSCs are present in low frequency in a variety of adult tissues such as bone marrow, adipose tissue, dental pulp and various fetal sources, to name but a few, it is important to consider the different properties of such cells when choosing a source for MSCs for a specific therapeutic application.

Klingemann et al. [2] provide a concise overview of the differentiation and expansion potential as well as the gene and cytokine expression profile of MSCs from different tissue sources and discuss their current status of clinical application. Remarkably, although all covered by the umbrella term MSC, they demonstrate very distinct gene expression fingerprints and differentiation potentials for cells from different sources. Based on these observations, the authors predict that future MSC-based therapies will take advantage of the different properties to provide cell transplants tailored to different medical indications. In order to gauge the clinical benefit of any such interventions, we however will require standardized manufacturing protocols to be able to generate preclinical and clinical evidence of the respective therapeutic potential and side effects. In recent years, European directives have been established that regulate all stages of clinical development from donor selection and procurement of cells and tissues up to the marketing of advanced therapies.

While the recently published Advanced Therapies Medicinal Product (ATMP) regulation will apply to medicinal products that are either 'prepared industrially or manufactured by a method involving an industrial process', it is not yet clear to what extent MSCs from different tissue sources, generated for individual clinical indications, may be subject of these regulations. In this issue, Ineke Slaper-Cortenbach [3], who is chair of the ISCT legal and regulatory affairs (LRA) committee, summarizes current European legislation with relevance to multipotent MSCs for clinical applications. She discusses under which circumstances MSCs may be considered ATMP. The articles by Bieback et al. [4] and Brooke et al. [5] further extend the general view on European guidelines and give valuable hands-on information on how to establish good manufacturing practice (GMP) and good clinical practice (GCP) compliant protocols for the processing and clinical examination of MSC-based medicines. Bieback and colleagues [4] delineate the different steps of the manufacturing process and discuss essential in-process controls and release criteria. In an effort to minimize the risk for the transmission of spongiform encephalopathy (TSE), the authors have pioneered work to replace the most commonly used fetal bovine serum as a

\begin{tabular}{ll}
\hline KARGER & ๑ 2008 S. Karger GmbH, Freiburg \\
Fax +497614520714 & Accessible online at: \\
$\begin{array}{l}\text { E-mail Information@Karger.de } \\
\text { www.karger.com }\end{array}$ & www.karger.com/tmh
\end{tabular}


media supplement by human derivatives. They suggest pooled human platelet lysate, pooled human blood type $\mathrm{AB}$ serum, and thrombin-activated platelet releasate in plasma as suitable alternatives. Brooke et al. [5] share with us their abundant experience in designing MSC based clinical trials and provide a highly informative review on collection and expansion protocols and their impact on efficacy and patient safety. They further point out critical safety parameters which should be addressed in phase I trials employing MSCs. In these, the primary focus should be safety endpoints such as acute infusional toxicity, bacterial infection, acceleration of pre-established malignancies, as well as the occurrence of ectopic tissue formation. Brooke et al. [5] also discuss different patient delivery procedures, cell doses, cryopreservation, and thawing protocols. Indeed all of these may be critical for the therapeutic potential of MSCs, but in turn may also have detrimental effects on cell quality and potency.

Both the regenerative and the immunosuppressive potential of MSCs appear to rely on their capacity to home, at least transiently, to the site of action. Thus, the knowledge of factors that may impact the homing of clinical applicable MSCs is of utmost importance. Therefore, in this issue Henschler et al. [6] review the current knowledge of mechanisms predicted to be relevant for the adhesion, migration, and homing competence of MSCs. Although primarily fibroblast-like cells, MSCs exhibit many of the mechanisms already described for the multistep interaction model during leukocyte recruitment through the vessel wall. Since an impairment of the complex receptor/ligand interactions involved in migration and homing of MSCs may adversely affect the outcome of MSC based clinical trials, Henschler et al. [6] postulate that investigational medical MSC products should be assayed for their migratory capacity. However, not only the manufacturing process and tissue source of MSCs may be critical with respect to their thera- peutic potency, but also factors that are inherent to the donor and/or patient of the initial tissue sample used for MSC isolation and expansion.

Lepperdinger et al. [7] give a comprehensive overview of the recent findings with special attention to age related changes within the MSC population and how these might impair their tissue regenerative capacity. This is of special importance since MSCs may be employed in an autologous fashion in heavily pretreated and elderly patients.

However, thus far, the most advanced clinical trials have been performed with ex vivo expanded allogeneic MSC in patients with steroid resistant GvHD where phase I [8] and phase II [9] trials have been successfully completed and appeared to be safe and effective in the treated cohort. Alan Tyndall [10] summarizes preclinical and clinical data available on the immunosuppressive action of MSCs in GvHD and various autoimmune diseases. While Tyndall points out that especially trials in GvHD have shown an acceptable benefit/risk ratio for MSC based therapies, he also stresses that the further development of such therapies demands an interdisciplinary approach. With regard to the clinical development of MSC based therapies which employ the immunosuppressive therapeutic effects, such interdisciplinary efforts are undertaken by the 'European League Against Rheumatism (EULAR)' and the 'European Group for Blood and Marrow Transplantation (EBMT)' [11].

As MSC based medicines further advance towards their clinical application, the standardization of manufacturing procedures and release criteria would certainly add to the development of the field as well as to the patients' safety. It is self-evident that our all combined and interdisciplinary efforts are needed to place MSC based products as valuable cellular therapies within the spectrum of modern regenerative approaches.

\section{References}

1 Horwitz EM, Le Blanc K, Dominici M, Mueller I, Slaper-Cortenbach I, Marini FC, Deans RJ, Krause DS, Keating A: Clarification of the nomenclature for MSC: the International Society for Cellular Therapy position statement. Cytotherapy 2005;5: 393-395.

2 Klingemann HG, Matzilecich D, Marchant J: Mesenchymal stem cells - sources and clinical applications. Transfus Med Hemother 2008;35(4):272-277.

$\checkmark 3$ Slaper-Cortenbach ICM: current regulations for the production of multipotent mesenchymal stroma cells for clinical application. Transfus Med Hemother 2008;35(4):295-298.

4 Bieback K, Schallmoser K, Klüter H, Strunk D Clinical protocols for the isolation and expansion of mesenchymal stromal cells. Transfus Med Hemother 2008;35(4):286-294.

\footnotetext{
5 Brooke G, Rossetti, T, Ilic N, Murray P, Hancock S, Pelekanos R, Atkinson K: Points to consider in designing mesenchymal stem cell-based clinical trials. Transfus Med Hemother 2008;35(4):279-285.

6 Henschler R, Deak E, Seifried E: Homing of mesenchymal stem cells. Transfus Med Hemother 2008; 35(4):306-312.

7 Lepperdinger G, Brunauer R, Gassner R, Jamnig A, Kloss F: Changes of the functional capacity of mesenchymal stem cells due to aging or age-associated disease - implications for clinical applications and donor recruitment. Transfus Med Hemother 2008;35(4):299-305.

8 Le Blanc K, Rasmusson I, Sundberg B, Gotherstrom C, Hassan M, Uzunel M, et al: Treatment of severe acute graft-versus-host disease with third party haploidentical mesenchymal stem cells. Lancet 2004;363:1439-1441.
}

$\checkmark 9$ Le Blanc K, Frassoni F, Ball L, Locatelli F, Roelofs $\mathrm{H}$, Lewis I, et al: Mesenchymal stem cells for treatment of steroid-resistant, severe, acute graft-versushost disease: a phase II study. Lancet 2008;371: 1579-1586.

10 Tyndall A: Multipotent mesenchymal stromal cells for autoimmune disease. Transfus Med Hemother 2008;35(4):313-318.

11 Tyndall A, Walker U, Cope A, Dazzi F, De Bari C, Fibbe W, et al: Immunomodulatory properties of mesenchymal stem cells - a review based on an interdisciplinary meeting held at the Kennedy Institute of Rheumatology Division, London, UK, 31 October 2005. Arthritis Res Ther 2007;9:301. 